\title{
Decrypting commensurate modulation, superstructure and inversion domain boundary in bismuth transition metal oxide through transmission electron microscopy
}

Satyam Choudhury ${ }^{1}$, Vishnumahanthy Mohan ${ }^{2}$, Hriddhi Ghosh ${ }^{3}$, Avnish Pal $^{1}$, Manish Singh $^{4}$, Rajiv Mandal $^{5}$ and Joysurya Basu ${ }^{5}$

${ }^{1}$ Department of Metallurgical Engineering IIT (BHU), Varanasi, Uttar Pradesh, India, ${ }^{2}$ Department of Metallurgical Engineering, IIT (BHU), visakhapatnam, Andhra Pradesh, India, ${ }^{3}$ Department of Metallurgical Engineering, IIT (BHU), United States, ${ }^{4}$ University of Connecticut, Connecticut, United States, ${ }^{5}$ Department of Metallurgical Engineering, IIT (BHU), Varanasi, Uttar Pradesh, India

Bismuth transition metal oxides (BMO, $\mathrm{M}=\mathrm{Cr}$, Mn) gained immense importance as functional materials due to their multiferroic properties [1]. In B-M-O ternary phase diagram $\mathrm{BiCrO} 3$ and $\mathrm{BiMnO} 3$ phases have been reported to be high pressure compounds [2], which are unstable at ambient temperature and pressure condition and transform to new structure with little variation in chemistry. This response is preferentially tuned by compositional fluctuation of one of its constituent element to impose changes onto sub-lattice of other two constituent elements, leading to redistribution of the atoms in 3D space. Phases specific to these systems exhibit wide range interesting functional properties such as fast oxygen ion conduction [3], candidate oxygen sensor material [4], etc. The motivation of this work is to elucidate the underlying short range order pervading through the bulk phase in $\mathrm{Bi}-\mathrm{Cr}-\mathrm{O}$ and $\mathrm{Bi}-\mathrm{Mn}-\mathrm{O}$ compounds.

Synthesis of Bi-Cr-O \& Bi-Mn-O compounds were carried out through solid state and wet chemical synthesis route. Characterization of synthesised samples was carried out by X-Ray diffraction (XRD) and Transmission Electron Microscopy (TEM), which enabled us to correlate studies at different length scale implementing bulk and statistical sampling methods. XRD studies validated the presence of orthorhombic Bi10Cr2O21, $\mathrm{Bi6Cr} 2 \mathrm{O} 15$, and BiMnO3 phases, which has been reported earlier $[5,6]$.

Selected area electron diffraction (SAED) of appropriate interaction volume through systematic tilting facilitates us to validate the structure and commensurate modulation in $\mathrm{Bi} 10 \mathrm{Cr} 2 \mathrm{O} 21, \mathrm{Bi} 6 \mathrm{Cr} 2 \mathrm{O} 15$ phases by completing Mobius triangle [7]. Extent of modulation along different crystallographic directions has been determined. In $\mathrm{Bi} 10 \mathrm{Cr} 2 \mathrm{O} 21$ compound, orientation relationship between the basic and the modulated unit have been established by overlaying simulated stereograms oriented along [-111] zone axis (ZA) of basic and [-233] ZA of modulated unit, subsequently the model is approximately verified. Diffraction contrast imaging (DCI) of modulated unit illustrates lattice fringes with complementary bright and dark fringe contrast arising from two distinct domain demarcated with inversion domain boundary (IDB) parallel to (200) modulated plane. DCI imaging of Bi6Cr2O15 phase over wide range of magnification reveals the signature of stripe contrast, which is comprised of five commensurately modulated units oriented along [001] ZA. The modulated unit comprised of four modulating units which can be distinguished in terms of their orientation, arrangement of atoms or both. This modulated unit can be imaged as fringes with alternate bright and dark contrast separated by $180^{\circ}$ IDB parallel to (020) modulated plane.

Electron diffraction with varying probe size coupled with DCI and high resolution phase contrast microscopy on distorted $\mathrm{BiMnO} 3$ phase confirms the evolution of superstructure, with dimension 8ax8c along [010] ZA. In addition, assembly of $4 \mathrm{a} \times 4 \mathrm{c}$ square nano domains have been observed along [010] (ZA) which mutually undergone in-plane rotation with extensive distortion along their interfaces. 


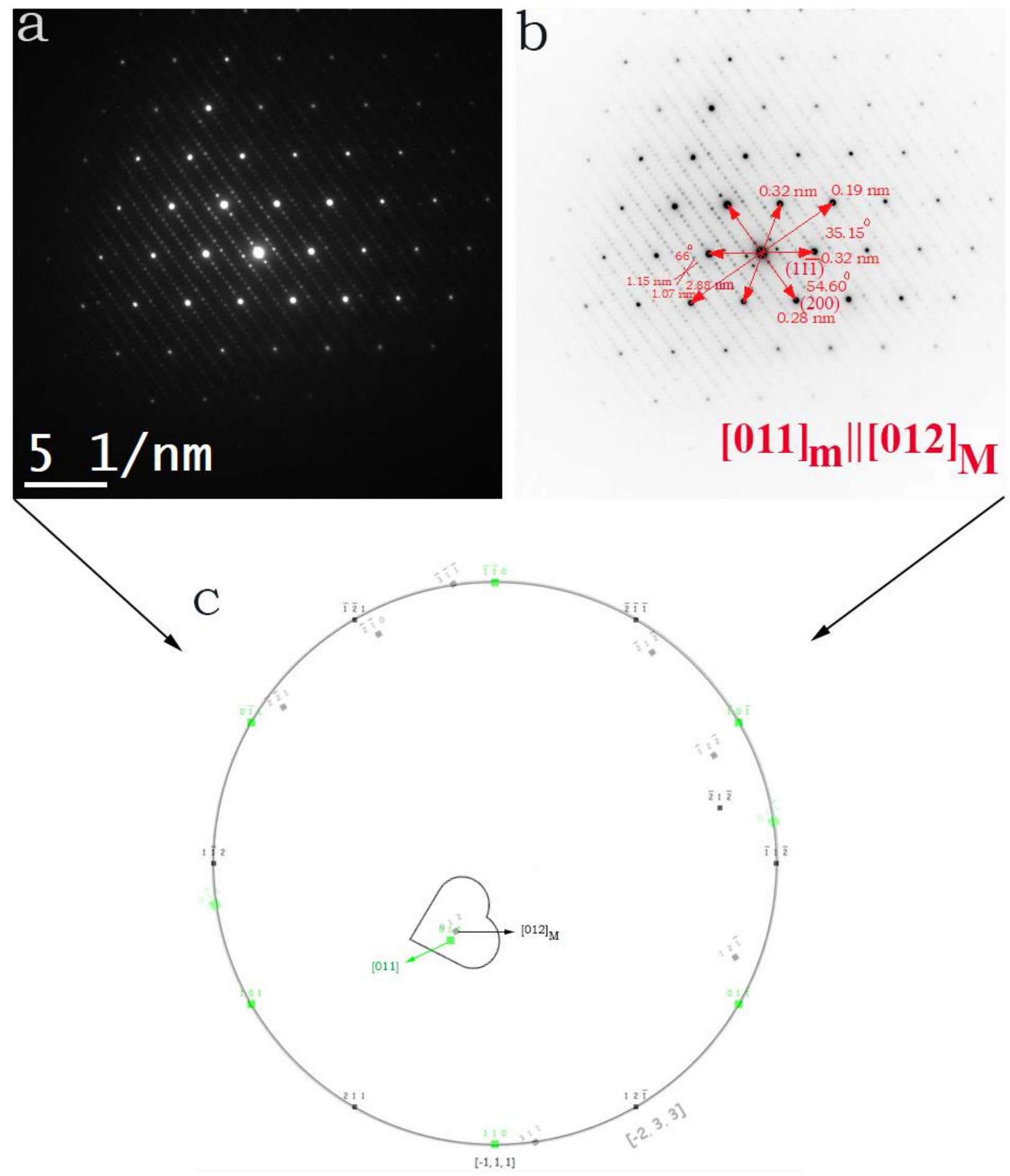

Figure 1. (a) Diffraction pattern decorated with clear signature of basic and modulated unit (b) [011] ZA of basic unit is oriented parallel to [012]M ZA of modulated unit represents a clear case of commensurate modulation. (c) The simulated model defining the orientation relationship between basic and modulated unit have been approximately verified. 


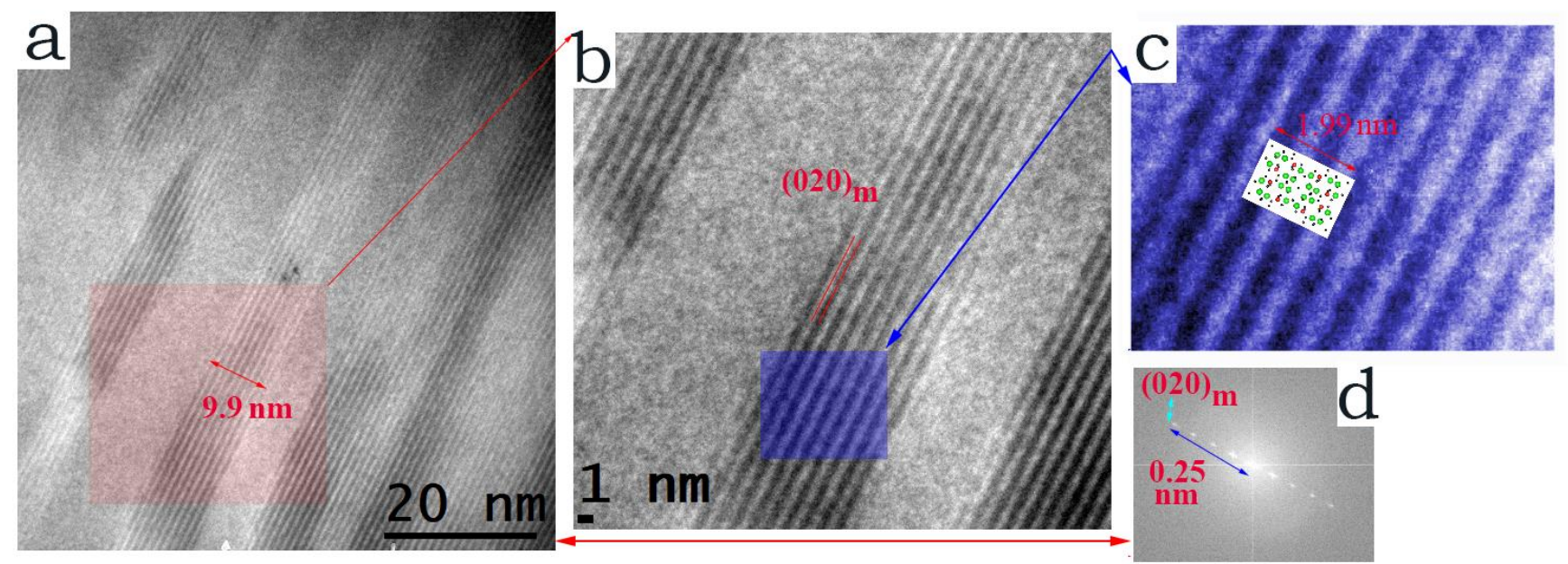

Figure 2. (a) Stripe contrast comprised of 20 fringes with alternate bright/dark contrast, thickness of each stripe equals $9.9 \mathrm{~nm}$ while inspecting along [001] zone axis. (b) Magnified image of stripes (c) Commensurately modulated unit comprised of four distinct units separated by $180^{\circ}$ Inversion domain boundary parallel to (020)M (d) corresponding Fast Fourier transformed image.

\section{References}

[1] Hill et al., J. Phys. Chem. B, vol. 106, no. 13, pp. 3383-3388, 2002.

[2] Sugawara et al., Journal of the Physical ..., vol. 25. pp. 1553-1558, 1968.

[3] Warda et al., J. Solid State Chem., vol. 149, no. 2, pp. 209-217, 2000.

[4] Meera et al., J. Nucl. Mater., vol. 487, pp. 174-185, 2017.

[5] Grins et al., J. Solid State Chem., vol. 163, no. 1, pp. 144-150, 2002.

[6] Calestani et al., Inorganic chemistry 53, no. 16 (2014): 8749-8754.

[7] D. B. Williams and C. B. Carter, Transmission Electron Microscopy, vol. 5, no. 721. 2009.

[8] The authors would like to acknowledge the financial support from UGC-DAE-CSR by the award number CSR-KN/CRS-94/2017-18/282.

[9] The author would like to acknowledge the support from Department of Science \& Technology inspired FIST programme. 